\title{
Surgical management in retroperitoneal fibrosis: beyond ureterolysis
}

\author{
Vigneswara Srinivasan Sockkalingam Venkatachalapathy ${ }^{*}$ (D) Datson George Palathullil, \\ Dempsey Mohan Sam and George Palathullil Abraham
}

\begin{abstract}
Background: Retroperitoneal fibrosis can be associated with bilateral dense and extensive periureteral adhesions. When ureterolysis could not be successfully performed due to disease extent and severity, elaborate ureteral reconstructive procedures will be required.

Case presentation: A young male with retroperitoneal fibrosis presented with bilateral hydroureteronephrosis. The ureteral involvement was extensive and adhesions were dense. He was managed by laparoscopic boari flap ureteroneocystostomy on one renal unit and laparoscopic nephrectomy with renal autotransplantation on the other renal unit.

Conclusions: Ureterolysis is not feasible in all cases of retroperitoneal fibrosis. Extensive bilateral ureteral reconstruction without using intestinal segments is feasible. Minimally invasive surgical reconstructive procedures can be successfully employed in such scenarios also.
\end{abstract}

Keywords: Retroperitoneal fibrosis, Laparoscopic boari flap, Renal autotransplantation

\section{Background}

Management of retroperitoneal fibrosis (RPF) with dense periureteral adhesions and bilateral extensive ureteral involvement is a challenging case scenario. Ureterolysis cannot be successfully performed in all such cases [1]. Boari flap, renal autotransplantation and ileal replacement of ureter are treatment options in such scenario. Ours is a case report of such extensive retroperitoneal fibrosis, where the patient was managed surgically. The uniqueness of our case report was the successful employment of minimally invasive methods and management without using bowel segments.

\section{Case presentation}

A 19-year-old male presented to us with the complaints of bilateral loin pain and weight loss of three months duration. He was under treatment from elsewhere for the

\footnotetext{
*Correspondence: viwasri@gmail.com
}

VPS Lakeshore Hospital, Kochi 682040, India complaints of ankle pain. He had been provisionally diagnosed as seronegative rheumatoid arthritis and had completed 8 months course of corticosteroid therapy. There was no history of prior abdominal surgery, radiation or other drugs intake. His serum creatinine at the time of presentation was $2 \mathrm{mg} / \mathrm{dl}$. Chest X-ray was normal. Ultrasonography showed bilateral hydroureteronephrosis (HUN).

Further evaluation with magnetic resonance urogram (MRU) showed bilateral HUN with clubbed calyces (Fig. 1a). Medial deviation of both ureters was noted at L4-L5 level. Also narrowing of ureters was noted at L4L5 level with periureteral stranding and beaded appearance of left ureter. Computerized tomography (CT) correlation did not show any ureteral calculus. Serum IgG4 levels were not significantly elevated. Antinuclear antibodies, rheumatoid factor, antineutrophil cytoplasmic antibodies, anti-thyroid peroxidase antibodies were negative. His urine smear and culture for acid fast bacilli (AFB) were negative. GeneXpert MTB/RIF (GeneXpert) was negative in urine sample [2]. 


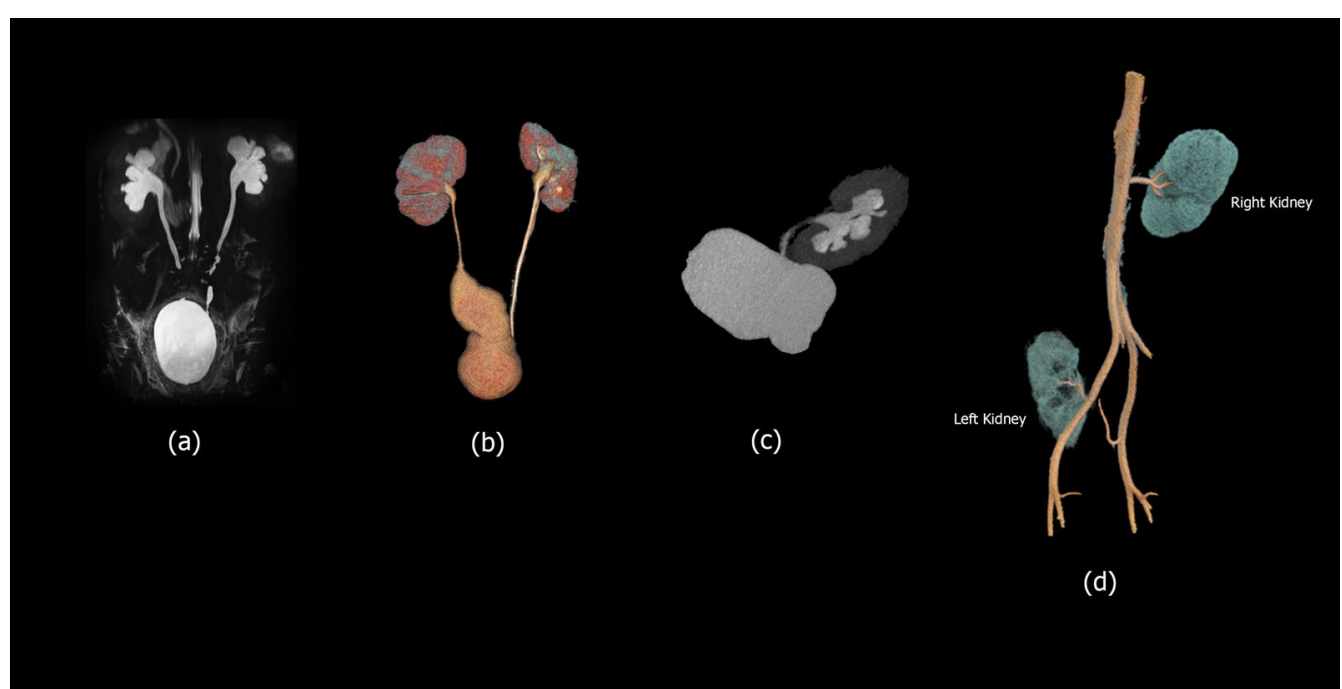

Fig. 1 a MRI showing bilateral hydroureteronephrosis with clubbed calyces. b CT showing unobstructed drainage in right renal unit. c CT showing unobstructed drainage in left autotransplanted renal unit. $\mathbf{d}$ CT images showing bilateral renal vascular tree after reconstructive procedures

The patient underwent cystoscopy, retrograde pyelogram (RGP) and bilateral double-J stent (DJS) placement. The cystoscopy showed normal capacity urinary bladder with diffuse cystitis changes and bilateral patulous ureteral orifices. RGP on both sides showed HUN with narrow and beaded lower ureters. The serum creatinine reached the level of $1.4 \mathrm{mg} / \mathrm{dl}$ after ureteral stenting.

The case was then discussed at the multidisciplinary departmental meeting. Since the patient had presented while he was already on steroid therapy and RGP findings were suspicious of genitourinary tuberculosis (GUTB), a decision to start antitubercular therapy (ATT) empirically was made (even though there was no confirmation of the diagnosis). We preferred 6 months course of ATT as it is suggested to be effective [3]. ATT was started and was continued for 6 months and the patient also improved symptomatically. DJS removal was performed after 6 months. After DJS removal, again serum creatinine reached the level of $3.6 \mathrm{mg} / \mathrm{dl}$. So DJS was replaced bilaterally.

One month after DJS replacement, serum creatinine level was $1.5 \mathrm{mg} / \mathrm{dl}$. Bilateral laparoscopic ureterolysis was planned electively. Reconstruction of the right renal unit was planned first as the right renal unit was considered the better functioning renal unit. Although differential renal function was not determined by radionuclide studies, MRU showed more renal parenchymal thickness on the right kidney.

Initially, laparoscopic right ureterolysis was attempted. Due to severe adhesions around the ureter distal to iliac vessel crossing, ureter could not be intactly mobilized (Fig. 2a) and laparoscopic boari flap

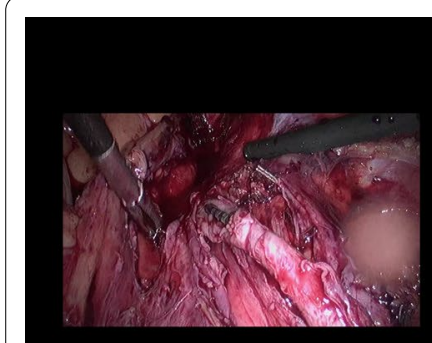

(a)

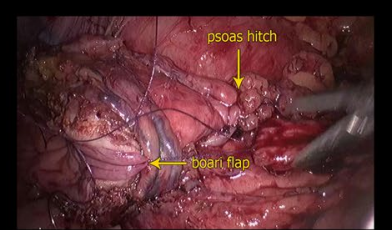

(b)

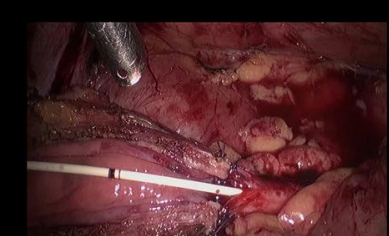

(c)

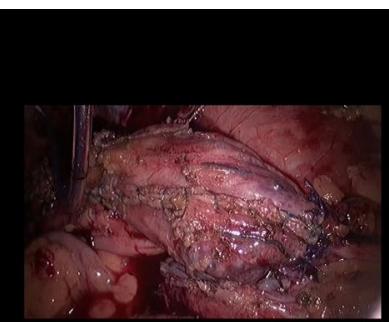

(d)

Fig. 2 a Dense right periureteral adhesions resulting in partial transection of ureter during attempted ureterolysis. b Boari flap with psoas hitch. $\mathbf{c}$ After performing posterior layer of boari flap ureteroneocystostomy. $\mathbf{d}$ After completion of boari flap ureteroneocystostomy 
ureteroneocystostomy with psoas hitch was performed (Fig. 2b-d). The histopathological examination (HPE) report of lower periureteral tissue taken for biopsy was suggestive of fibrofatty tissue with chronic inflammation. There was no evidence of granuloma in the biopsy specimen. The immunohistochemistry (IHC) ruled out IgG4related disease. The right DJS removal was performed 3 months after the surgery.

Three months after right DJS removal left laparoscopic ureterolysis was attempted. We contemplated the possibility of difficulty in dissection and we had planned to proceed with renal autotransplantation if we could not successfully proceed with laparoscopic ureterolysis. Preoperative CT had depicted unobstructed urinary drainage in right renal unit (Fig. 1b) and single left renal artery and vein. Intraoperatively, severe periureteral adhesions (Fig. 3a) starting from the level of iliac vessel crossing hindered the progression of ureterolysis and renal autotransplantation had to be performed. Renal autotransplantation was preferred over boari flap on the left side, as the bladder capacity and perivesical adhesions would not have allowed bilateral boari flap ureteroneocystostomy. After laparoscopic nephrectomy, graft kidney was retrieved via a pfannenstiel incision. Renal bed was prepared via a left modified gibson incision. Renal artery and vein were anastomosed to the external iliac artery and vein, respectively (Fig. 3b, c). Ureteroneocystostomy was performed with healthy proximal ureter (Fig. 3d).

Biopsies had been taken from lower periureteral tissues and histopathological examination revealed reactive lymph nodes with background fibrosis. No plasmacytosis or evidence of chronic inflammatory reaction was seen. IHC features were also consistent with reactive changes. The left DJS removal was performed 6 weeks after left renal autotransplantation.
CT urogram performed 3 months after left ureteral stent removal showed unobstructed drainage pattern on left kidney (Fig. 1c) and enhancement of renal parenchyma was noted on the autotransplanted kidney (Fig. 1d). Six months after left DJS removal, patient's creatinine was $1.3 \mathrm{mg} / \mathrm{dl}$ and the patient was asymptomatic. The patient was not on any medications at that time.

\section{Discussion}

Symptoms of RPF are usually non-specific in the initial stages. The ureters are the primary organs affected in the later stages of retroperitoneal fibrosis and bilateral involvement is more common than unilateral involvement [1]. The secondary causes of RPF may be seen in one third of the cases [1].

Corticosteroids are the mainstay in medical treatment and optimal regimen is not well defined [4]. In our case, the disease presentation had happened while the patient was already on corticosteroids and the initial imaging findings raised the suspicion GUTB. Also, since there are case reports of tuberculosis triggering retroperitoneal fibrosis [5], we had started ATT. Although the patient had symptomatic improvement, no evidence of tuberculosis could be found on the HPE of the biopsy specimen.

In a large series of 204 patients, ureterolysis was the most common surgical intervention performed [1]. But our patient had dense and extensive distal periureteral adhesions and ureter could not be freed. As a result, we had to perform the aforementioned procedures. Ureteral reconstruction using bowel segments may cause many electrolyte and metabolic abnormalities [6]. Considering this fact and the age of the patient, we avoided using intestinal segments in ureteral reconstruction.

To our knowledge ours is the first case report of extensive retroperitoneal fibrosis which is managed by laparoscopic boari flap ureteroneocystostomy on one renal unit

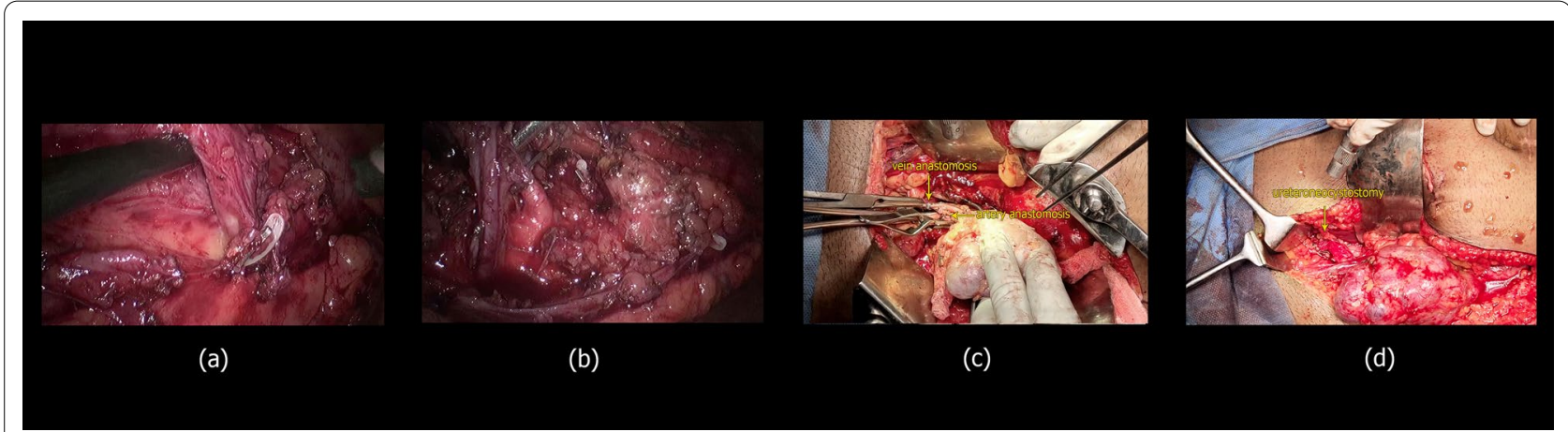

Fig. 3 a Dense left periureteral adhesions from the level of iliac vessel crossing. $\mathbf{b}$ Left single renal artery and vein dissected out. c After completion of vascular anastomosis during renal autotransplantation. $\mathbf{d}$ After completion of ureteroneocystostomy during renal autotransplantation 
and laparoscopic nephrectomy with renal autotransplantation on the other renal unit.

\section{Conclusions}

Retroperitoneal fibrosis with HUN not responding to medical treatment, may warrant surgical correction and ureterolysis may not be feasible in all cases. Extensive bilateral ureteral reconstruction without using intestinal segments may suffice. Minimally invasive treatment options can be used successfully in these scenarios also.

\section{Abbreviations}

AFB: Acid fast bacilli; ATT: Antitubercular therapy; CT: Computerized tomography; DJS: Double J stent; GUTB: Genitourinary tuberculosis; HPE: Histopathological examination; IHC: Immunohistochemistry; MRU: Magnetic resonance urography; RGP: Retrograde pyelogram; RPF: Retroperitoneal fibrosis.

\section{Acknowledgements}

Mr Bijoy (visual editor), for editing the photographs.

\section{Authors' contribution}

VSV contributed in concept, design, literature search, manuscript preparation and manuscript review. DGP contributed in design, manuscript editing and manuscript review. DMS contributed in manuscript editing and manuscript review. GPA contributed in operating the patient, manuscript editing and manuscript review. All authors have read and approved the final manuscript.

\section{Funding}

None.

Availability of data and materials

Not applicable.

\section{Declarations}

Ethics approval and consent to participate At our institute not needed for this case report.

\section{Consent for publication}

Written informed consent was obtained from the patient for publication of this case report and accompanying images.

\section{Competing interests}

The authors declare that they have no competing interests.

Received: 23 March 2021 Accepted: 4 August 2021

Published online: 16 August 2021

\section{References}

1. Brandt AS, Kamper L, Kukuk S, Haage P, Roth S (2011) Associated findings and complications of retroperitoneal fibrosis in 204 patients: results of a urological registry. J Urol 185(2):526-531

2. Pang Y, Shang Y, Lu J, Liang Q, Dong L, Li Y et al (2017) GeneXpert MTB/RIF assay in the diagnosis of urinary tuberculosis from urine specimens. Sci Rep 7:6181

3. Kadhiravan T, Sharma SK (2008) Medical management of genitourinary tuberculosis. Indian J Urol 24(3):362-368

4. Pipitone N, Vaglio A, Salvarani C (2012) Retroperitoneal fibrosis. Best Pract Res Clin Rheumatol 26(4):439-448

5. Greco P, Vaglio A, Corradi D, Cobelli R, Zompatori M, Buzio C (2005) Tuberculosis as a trigger of retroperitoneal fibrosis. Clin Infect Dis 41(7):e72-e75

6. Hansen MH, Hayn M, Murray P (2016) The use of bowel in urologic reconstructive surgery. Surg Clin 96(3):567-582

\section{Publisher's Note}

Springer Nature remains neutral with regard to jurisdictional claims in published maps and institutional affiliations.

\section{Submit your manuscript to a SpringerOpen ${ }^{\circ}$ journal and benefit from:}

- Convenient online submission

- Rigorous peer review

- Open access: articles freely available online

- High visibility within the field

- Retaining the copyright to your article

Submit your next manuscript at $\boldsymbol{\nabla}$ springeropen.com 